\title{
REGULARITY PROPERTIES OF FREE DISCONTINUITY SETS
}

\author{
Francesco MADDALENA, Sergio SOLIMINI \\ Dipartimento di Matematica, Politecnico di Bari, Italy \\ Received 22 May 2000, revised 26 February 2001
}

ABSTRACT. - This paper is concerned with the problem of estimating the dimension, expected to be $N-2$, of the singular set of a minimizer of a functional with free discontinuities in $N$ dimensions. The best result already known, namely the $(N-1)$-negligibility, is improved here. (C) 2001 L'Association Publications de l'Institut Henri Poincaré. Published by Elsevier B.V. All rights reserved

RÉSUMÉ. - On s'attend à ce que la dimension de l'ensemble singulier d'un minimum d'une fonctionnelle à discontinuité libre soit $N-2$. Selon le meilleur résultat connu jusqu'à présent, cet ensemble est $N-1$ négligeable. Nous améliorons ce résultat.

(C) 2001 L'Association Publications de l'Institut Henri Poincaré. Published by Elsevier B.V. All rights reserved

\section{Introduction}

This paper is concerned with the regularity properties of a singularity set $K$ minimizing the Mumford-Shah functional. This functional was introduced in [18] and we refer the reader to the literature on the subject (see the articles [1-3,6-11] and the books $[5,16])$. We recall that a minimum of this functional is represented by a pair $(u, K)$ constituted by a function variable $u$ and a set variable $K$. We deal with the problem of establishing that the set of points where $K$ is not locally a $C^{1}$ surface (the singularity set of $K$ ) is small. It seems reasonable to expect that such a set is in general not empty and has Hausdorff dimension $(N-2)$ but, as far as we know, this has not been proved. The best result in this direction is due to L. Ambrosio, N. Fusco and D. Pallara [4] (and in dimension two also indipendently by G. David in [8]) who show that the singularity set of $K$ is $\mathcal{H}^{N-1}$-negligible and this constitutes a first step in the above perspective. Such a result is reached through a regularity technique which gives a monotonicity estimate starting from suitable balls (in which the scaled flatness and the scaled Dirichlet integral are smaller than a given threshold) and it is easy to check that almost every point in $K$ is the center of a good ball.

In this paper, by recovering the techniques exposed in [13] and the a priori estimates of [20], we prove that every ball centered on $K$ contains, at an estimable scale 
transition, a sub-ball in which $K$ is a $C^{1}$ surface splitting approximatively the ball in two hemispheres. On each one of the two hemispheres the oscillation (normalized with respect to the scale) of the function $u$ is small and between them the (normalized) jump of $u$ is large. The regularity of $K$ is obtained through an application of the monotonicity results of [4], whose initial hypotheses are assured by the previously employed techniques. Such a result can be considered as a further step towards the estimate of the dimension of the singular part of $K$ because it allows the application of [10, Lemma 5.8] which in turn shows that such a dimension is strictly lower than $N-1$.

The results of this paper were announced at the Meeting on Differential Equations and Calculus of Variations held at Isola d'Elba in October 1999. In such occasion we knew from L. Ambrosio that an analogous result was obtained by S. Rigot [19] and that it matches with the hypotheses of [10, Lemma 5.8].

\section{Notation and main results}

Let $\Omega \subset \mathbb{R}^{N}$ be an open set and let $g$ be a given measurable function from $\Omega$ in $[0,1]$. In this paper we study some regularity properties concerning the minima of the functional with free discontinuities

$$
E(u, K)=J(u, K)+\mathcal{H}^{N-1}(K),
$$

where $J(u, K)$ denotes the value of the elliptic functional

$$
J(u, K)=\int_{\Omega \backslash K}|\nabla u|^{2}+\int_{\Omega \backslash K}|g-u|^{2}
$$

and $\mathcal{H}^{N-1}(K)$ is the $(N-1)$-dimensional Hausdorff measure of $K$. An admissible pair $(u, K)$ is defined as a pair with $u \in L_{l o c}^{1}(\Omega)$ and $K$ varying among the closed subsets of $\Omega$. When $K$ is given, one can determine a unique function $u(K)$ which minimizes $J$ on the open set $\Omega \backslash K$, therefore one can regard the functionals $E$ and $J$ as only depending on the set variable $K$. We shall study some regularity properties of the sets $K$ minimizing $E$. If $K$ is a minimum of $E, u=u(K)$ and $B \subset \Omega$ is any ball of radius $R \leqslant 1$, then (see [20])

$$
\int_{B}|\nabla u|^{2}+\mathcal{H}^{N-1}(K \cap B) \leqslant|B|+\mathcal{H}^{N-1}(\partial B) \leqslant(N+1) b_{N} R^{N-1},
$$

where $b_{k}$ denotes the measure of the unit ball of $\mathbb{R}^{k}$. The regularity of an optimal set $K$ is expressed in terms of some geometric properties which we are going to introduce. Let $X \subset \mathbb{R}^{N}$ be an open set and $u \in L_{l o c}^{1}(X)$ be a real function. For a given positive constant $c$, the following weak summability condition states that the distributional gradient $\nabla u$, extended by zero on $\mathbb{R}^{N} \backslash X$, belongs to the Morrey Space $L^{1,(2 N-1) / 2}$. When $X \subset \Omega$ and $u$ is a minimum of the Mumford-Shah functional, it trivially follows from (1.3) which 
actually implies that $\nabla u \in L^{2, N-1}$. It is worth to remark that this is a weaker case of $L^{2 N}$-summability.

$$
\text { For every ball } B \subset \mathbb{R}^{N}: \int_{B}|\nabla u| \leqslant c|B|^{\frac{2 N-1}{2 N}} \leqslant\|u\|_{2 N}^{*} R^{\frac{2 N-1}{2}},
$$

where $R$ is the radius of $B$. Now we are going to introduce some definitions which will be employed in characterizing the local behavior of the set $K$. If $u$ is any real function defined on a subset $X$ of $\mathbb{R}^{N}$, then $\operatorname{osc}_{X} u$ will represent the oscillation of $u$ on $X$, that is $\operatorname{osc}_{X} u=\sup _{X} u-\inf _{X} u$.

Definition 1.1. - Let $\varepsilon>0$ be given. A ball $B$ of radius $R$ is said to be $\varepsilon$-split by a function $u: B \rightarrow \mathbb{R}$ if it does not contain any subset $\tilde{B}$ such that

$$
\begin{gathered}
|\tilde{B}| \geqslant\left(\frac{1}{2}+\varepsilon\right)|B|, \\
\operatorname{osc}_{\tilde{B}} u \leqslant \varepsilon^{-1} R^{1 / 2} .
\end{gathered}
$$

Definition 1.2. - The ball $B$ of radius $R$ is said to be $\varepsilon$-split by $K$ if there exists $u: B \rightarrow \mathbb{R}$, satisfying (WS) with $\|u\|_{2 N}^{*}=1$ on $B \backslash K$, such that $B$ is $\varepsilon$-split by $u$.

Let $B$ be a given ball with radius $R$, for every $\varepsilon>0$ we shall denote by $\mathcal{S}_{\varepsilon}(B)$ the set of all closed subsets $K$ of $B$ such that $B$ is $\varepsilon$-split by $K$. We put

$$
s_{\varepsilon}(B)=\inf _{K \in \mathcal{S}_{\varepsilon}} \mathcal{H}^{N-1}(K \cap B), \quad s(\varepsilon)=s_{\varepsilon}\left(B^{1}\right),
$$

where $B^{1}$ denotes the unitary ball of $\mathbb{R}^{N}$, and

$$
s_{0}=\lim _{\varepsilon \rightarrow 0} s(\varepsilon) .
$$

We note that $s(\varepsilon)$ is a monotone decreasing function and that

$$
s_{0}=\sup _{\varepsilon>0} s(\varepsilon) \leqslant b_{N-1} .
$$

By rescaling, we can easily see that for every $R>0$

$$
s_{\varepsilon}(B)=R^{N-1} s(\varepsilon) .
$$

We shall say that $K$ satisfies the Bisection Property when for every $\varepsilon>0$ there exists $\alpha(\varepsilon)>0$ such that, for every ball $B \subset \Omega$ centered on $K$, with radius $R \leqslant 1$, there exists a $\varepsilon$-split ball $B^{\prime} \subset B$ with a radius $r \geqslant \alpha(\varepsilon)$. Given a ball $B$ centered on $K$, with a radius $R$, we say that the set $K$ is $\varepsilon$-concentrated on $B$, if the mean density of $K$ on $B$ is bigger than $1-\varepsilon$, namely

$$
\mathcal{H}^{N-1}(K \cap B)>(1-\varepsilon) b_{N-1} R^{N-1} .
$$


We shall say that $K$ satisfies the Concentration Property when for every $\varepsilon>0$ there exists $\alpha(\varepsilon)>0$ such that, for every ball $B$ centered on $K$, with radius $R \leqslant 1$, there exists a ball $B^{\prime} \subset B$ with radius $r \geqslant \alpha(\varepsilon)$ where $K$ is $\varepsilon$-concentrated. In [13] it has been proved that the inequality in (1.6) is actually an equality. This is equivalent to say that for every $\varepsilon>0$ there exists $\delta$ such that, if the ball $B$ is $\delta$-split by the set $K$, then $K$ turns out to be $\varepsilon$-concentrated on $B$. In particular, this shows that the Concentration Property follows from the Bisection Property. Furthermore, from the Concentration Property, the Uniform Density Property trivially follows, namely there exists a positive constant $\beta$ such that, if $B$ is a given ball centered at a point of $K$, of radius $R \leqslant 1$, then

$$
\mathcal{H}^{N-1}(B \cap K) \geqslant \beta R^{N-1} .
$$

In the case of sets $K$ which minimize $E$, the bisection property is proved in [20]. Finally, we introduce the following quantities which play a key role in studying the regularity of the set $K$ in the ball $B=B(x, r)$ (see [4] and [5] for more details).

Scaled flatness

$$
\mathbf{A}_{K}(B)=\mathbf{A}_{K}(x, r)=r^{-N-1} \min _{P \in \mathcal{A}} \int_{B \cap K} d^{2}(y, P) \mathrm{d} \mathcal{H}^{N-1},
$$

where $\mathcal{A}$ denotes the set of affine hyperplanes in $\mathbb{R}^{N}$. $\mathbf{A}_{K}(B)$ measures the scaled flatness of $K$ into the ball $B$.

Scaled tilt

$$
\mathbf{T}_{K}(B, T)=\mathbf{T}_{K}(x, r, T)=r^{1-N} \int_{B \cap K}\left\|S_{y}-T\right\|^{2} \mathrm{~d} \mathcal{H}^{N-1},
$$

where $T$ is a given hyperplane of $\mathbb{R}^{N}$ and $\left\|S_{y}-T\right\|$ denotes the Grassmann distance of the approximate tangent space $S_{y}$ to $K$ at $y$ from $T$. It measures the oscillation of $S_{y}$ in $B$ with respect to a given hyperplane $T$.

Scaled Dirichlet integral

$$
\mathbf{D}_{u}(B)=\mathbf{D}(x, r)=r^{1-N} \int_{B}|\nabla u|^{2} \mathrm{~d} y .
$$

Let $\varepsilon>0$, the closed $\varepsilon$-neighborhood of the set $X \subset \mathbb{R}^{N}$ is

$$
X(\varepsilon)=\left\{x \in \mathbb{R}^{N} \mid d(x, X) \leqslant \varepsilon\right\} .
$$

We shall denote by $\mathbf{d}(A, B)$ the Hausdorff distance between the sets $A$ and $B$. In the sequel we show how the bisection and concentration properties can be improved as stated in the following theorem which represents the main result of this paper.

THEOREM 1.1. - For every $\varepsilon>0$ there exists $\alpha(\varepsilon)$ such that, for every $B(x, R) \subset \Omega$ with $x \in K$ and $R \leqslant 1$ there exists a ball $B^{\prime}=B(y, r) \subset B(x, R)$ with radius $r \geqslant \alpha(\varepsilon)$ such that the following properties hold: 
(1) $B^{\prime}$ is $\varepsilon$-split.

(2) $\left|r^{1-N} \mathcal{H}^{N-1}\left(K \cap B^{\prime}\right)-b_{N-1}\right|<\varepsilon$.

(3) $\mathbf{A}_{K}\left(B^{\prime}\right)<\varepsilon$.

(4) There exists a hyperplane $P$ through the center of $B^{\prime}$ such that $\mathbf{T}_{K}\left(B^{\prime}, P\right)<\varepsilon$.

(5) $r^{-1} \mathbf{d}(K, P)<\varepsilon$, where $P$ is as above.

(6) $\mathbf{D}_{u}\left(B^{\prime}\right)<\varepsilon$.

(7) The set $K \cap B^{\prime}$ is a $C^{1,1 / 4}$ surface containing the center $y$ and dividing $B^{\prime}$ in two parts $\mathrm{H}_{1}$ and $\mathrm{H}_{2}$.

(8) For $i=1,2: \operatorname{osc}_{H_{i}} u<\varepsilon \sqrt{r}$.

(9) The jump of $u$ between $H_{1}$ and $H_{2}$ is greater than $\varepsilon^{-1} \sqrt{r}$.

The properties stated in the above theorem, excepted (7), are obtained through a fine application of the results of [13], by showing that the bisection property can be employed in such a way to get balls contained in the main ball, which are $\varepsilon$-split and enjoy suitable minimality conditions. Property (7) is obtained from the former ones as an immediate consequence of the regularity techniques of [4], which can be applied thanks to the properties (3) and (6). Moreover, by fixing a suitable value of $\varepsilon$, we have the following result.

COROLLARY 1.2. - There exists $\alpha>0$ such that for every $B(x, R) \subset \Omega$ with $x \in K$ and $R \leqslant 1$ there exists a ball $B^{\prime}=B(y, r) \subset B(x, R)$, with radius $r \geqslant \alpha R$ such that the set $S=K \cap B^{\prime}$ is a $C^{1,1 / 4}$ surface containing $y$.

\section{Proof of the results}

In [13] it is shown that, given a positive constant $\varepsilon^{\prime}>0$, one can determine two other constants $t, n \in \mathbb{N}$, which permit to state, for any positive $\delta$, the minimality condition

For every $\delta$-split ball $B^{\prime} \subset B$ of radius $r \geqslant t^{n} R$ :

$$
R^{1-N} \mathcal{H}^{N-1}(K) \leqslant\left(1+\frac{\varepsilon^{\prime}}{2}\right) r^{1-N} \mathcal{H}^{N-1}\left(K \cap B^{\prime}\right) \text {. }
$$

Let $\varepsilon$ be a given positive number. In [13] it is proved that one can subsequently determine another positive constant $\varepsilon^{\prime}(\varepsilon)$, which allows to write $\left(\mathrm{M}_{\delta}\right)$ in a form which depends on $\varepsilon$, in such a way to have the following statement [13, Corollary 6.1].

LEMMA 2.1. - For every $\varepsilon, \delta_{1}, c_{0}>0$, there exists $\delta_{2}>0$ such that, if $B$ is $\delta_{2}$-split by $K$, satisfies $\left(\mathrm{M}_{\delta_{1}}\right)$ and $\mathcal{H}^{N-1}(K \cap B) \leqslant c_{0} R^{N-1}$, then $\mathbf{A}_{K}(B)<\varepsilon$.

Since for the sets $K$ minimizing the Mumford-Shah functional the condition on $c_{0}$ is satisfied, by (1.3), when $c_{0}=(N+1) b_{N}$, for all the minimal sets $K$ we have in particular the following statement.

LEMMA 2.2. - For every $\varepsilon, \delta_{1}>0$, there exists $\delta_{2}=I\left(\delta_{1}\right)$ such that, if $B$ is $\delta_{2}$-split by $K$ and satisfies $\left(\mathrm{M}_{\delta_{1}}\right)$, then $\mathbf{A}_{K}(B)<\varepsilon$.

An iterated version of the above result will allow us to get free from Condition $\left(\mathrm{M}_{\delta_{1}}\right)$, by passing to a suitable sub-ball $B^{\prime}$ under a scale transition which can be estimated. 
LEMMA 2.3. - For every $\varepsilon>0$ there exists $\alpha(\varepsilon)>0$ such that every ball $B \subset \Omega$, centered on $K$, with radius $R \leqslant 1$, contains a ball $B^{\prime}$ centered on $K$, $\varepsilon$-split, with radius $r \geqslant \alpha(\varepsilon) R$ such that $\mathbf{A}_{K}\left(B^{\prime}\right)<\varepsilon$.

Proof. - Given $\varepsilon>0$, we fix $\varepsilon^{\prime}$ as above described. Let $m \in \mathbb{N}$ such that $\left(1+\frac{\varepsilon^{\prime}}{2}\right)^{m-1}>$ $(N+1) b_{N} s(\varepsilon)^{-1}$. We define a sequence $\delta_{1}, \delta_{2}, \ldots, \delta_{m}$ by taking $\delta_{1}=\varepsilon, \delta_{2}=I\left(\delta_{1}\right)$ as given by Lemma 2.2 and, in general, $\delta_{i}=I\left(\delta_{i-1}\right)$, where $I$ is as defined in Lemma 2.2 and $i=2, \ldots, m$. By virtue of [20, Theorem 3 (Bisection Property)] we can claim that there exists a constant $\beta=\beta\left(\delta_{m}\right)$ such that, if $B$ is as in the thesis, we can find a ball $B_{m} \subset B$ of radius $r_{m} \geqslant \beta R, \delta_{m}$-split by $K$. If such a ball satisfies $\left(\mathrm{M}_{\delta_{m-1}}\right)$, then $\mathbf{A}_{K}\left(B_{m}\right)<\varepsilon$. Otherwise, we can take $B_{m-1} \subset B_{m}$, with a radius $r_{m-1}>t^{n} r_{m}, \delta_{m-1}$-split by $K$ and such that

$$
r_{m-1}^{1-N} \mathcal{H}^{N-1}\left(K \cap B_{m-1}\right) \leqslant\left(1+\frac{\varepsilon^{\prime}}{2}\right)^{-1} r_{m}^{1-N} \mathcal{H}^{N-1}\left(K \cap B_{m}\right) .
$$

If the new ball $B_{m-1}$ satisfies $\left(\mathrm{M}_{\delta_{m-2}}\right)$, we stop. Otherwise, we can take $B_{m-2} \subset B_{m-1}$, with a radius $r_{m-2}>t^{n} r_{m-1}, \delta_{m-2}$-split by $K$ and such that

$$
\begin{aligned}
r_{m-2}^{1-N} \mathcal{H}^{N-1}\left(K \cap B_{m-2}\right) & \leqslant\left(1+\frac{\varepsilon^{\prime}}{2}\right)^{-1} r_{m-1}^{1-N} \mathcal{H}^{N-1}\left(K \cap B_{m-1}\right) \\
& \leqslant\left(1+\frac{\varepsilon^{\prime}}{2}\right)^{-2} r_{m}^{1-N} \mathcal{H}^{N-1}\left(K \cap B_{m}\right) .
\end{aligned}
$$

Then we iterate this process as far as we do not find a ball $B_{i}, i \geqslant 2$, which enjoys $\left(\mathrm{M}_{\delta_{i-1}}\right)$. We claim that this process cannot reach $i=1$. Indeed, at every step we have

$$
r_{i}^{1-N} \mathcal{H}^{N-1}\left(K \cap B_{i}\right) \leqslant\left(1+\frac{\varepsilon^{\prime}}{2}\right)^{i-m} r_{m}^{1-N} \mathcal{H}^{N-1}\left(K \cap B_{m}\right)
$$

So, if we could let $i=1$, we should find

$$
\begin{aligned}
r_{1}^{1-N} \mathcal{H}^{N-1}\left(K \cap B_{1}\right) & \leqslant\left(1+\frac{\varepsilon^{\prime}}{2}\right)^{1-m} r_{m}^{1-N} \mathcal{H}^{N-1}\left(K \cap B_{m}\right) \\
& <\left(1+\frac{\varepsilon^{\prime}}{2}\right)^{1-m}(N+1) b_{N}<s(\varepsilon),
\end{aligned}
$$

a contradiction since $B_{1}$ is found to be $\varepsilon$-split. The contradiction shows the claim and so we can take as $B^{\prime}$ the last ball $B_{i}$ given by the iteration process with $i \geqslant 2$, which is $\delta_{i}$-split, has a radius $r=r_{i} \geqslant t^{n(m-2)} r_{m} \geqslant t^{n(m-2)} \beta R$ and enjoys $\left(\mathrm{M}_{\delta_{i-1}}\right)$. So the property $\mathbf{A}_{K}\left(B^{\prime}\right)<\varepsilon$ follows by Lemma 2.2 , since $\delta_{i}=I\left(\delta_{i-1}\right)$, and we can finally take $\alpha(\varepsilon)=t^{n(m-2)} \beta\left(\delta_{m}\right)$.

LEMMA 2.4. - The ball $B^{\prime}$ found in the previous lemma can be also taken in such a way that there exists a hyperplane $P$ such that

$$
K \cap B^{\prime} \subset P(\varepsilon r)
$$


Proof. - Let $\beta$ be the density constant appearing in (1.8). We can apply the previous lemma in such a way to get a $\beta\left(\frac{\varepsilon}{2}\right)^{N+1}$-split ball $B^{\prime \prime}$, such that $\mathbf{A}_{K}\left(B^{\prime \prime}\right)<\beta\left(\frac{\varepsilon}{2}\right)^{N+1}$ and with a radius greater or equal to $\alpha\left(\beta\left(\frac{\varepsilon}{2}\right)^{N+1}\right) R$. The estimate on $\mathbf{A}_{K}\left(B^{\prime \prime}\right)$ means that there exists a hyperplane $P$ such that

$$
\mathbf{A}_{K}\left(B^{\prime \prime}\right)=r^{-N-1} \int_{B^{\prime \prime} \cap K} d^{2}(y, P) \mathrm{d} \mathcal{H}^{N-1}(y)<\beta\left(\frac{\varepsilon}{2}\right)^{N+1} .
$$

Now we take as $B^{\prime}$ the ball with the same center of $B^{\prime \prime}$ and radius $r=\left(1-\frac{\varepsilon}{2}\right) r^{\prime \prime}$ and we claim that $K \cap B^{\prime} \subset P(\varepsilon r)$. Indeed, otherwise, we can find $x \in K \cap B^{\prime}$ such that $d(x, P)>\varepsilon r$ and therefore $B\left(x, \frac{\varepsilon}{2} r\right) \subset B^{\prime \prime}$. By using (1.8) we find

$$
\begin{aligned}
\int_{B^{\prime \prime \cap K}} d^{2}(y, P) \mathrm{d} \mathcal{H}^{N-1}(y) & \geqslant \int_{B\left(x, \frac{\varepsilon}{2} r\right) \cap K} d^{2}(y, P) \mathrm{d} \mathcal{H}^{N-1}(y) \\
& \geqslant\left(\frac{\varepsilon}{2} r\right)^{2} \mathcal{H}^{N-1}\left(K \cap B\left(x, \frac{\varepsilon}{2} r\right)\right) \geqslant \beta\left(\frac{\varepsilon}{2} r\right)^{N+1},
\end{aligned}
$$

in contradiction to (2.10).

We briefly recall some arguments discussed in [13], which we are going to use in a simpler context. Let $P$ be as in the previous lemma, given $\varepsilon>0$ we take the two disks $D_{1}$ and $D_{2}$, obtained by cutting $B^{\prime}$ with the two hyperplanes parallel to $P$ and distant $\varepsilon$ from $P$, labeled so that $\mathcal{H}^{N-1}\left(D_{1}\right) \leqslant \mathcal{H}^{N-1}\left(D_{2}\right)$. Let $p_{1}$ denote the orthogonal projection onto the hyperplane containing $D_{1}$. Thus, the ball $B^{\prime}$ comes out divided in three regions, namely the set enclosed between $D_{1}$ and $D_{2}$, which will be called $F_{\varepsilon}$ and the remaining two regions $E_{1}$ and $E_{2}$. By Lemma 2.4, $K \cap\left(E_{1} \cup E_{2}\right)=\emptyset$ follows, so by Morrey's Hölder continuity theorem [17, Theorem 3.5.2] we know that the oscillation of $u$ on each set $E_{i}$ is bounded by $c \sqrt{r}$, where $c$ is a universal constant. Therefore, the $\varepsilon$-splitness of $B^{\prime}$ implies that for $i=1,2$ we have from (1.4)

$$
\left|E_{i}\right|<\left(\frac{1}{2}+\varepsilon\right)\left|B^{\prime}\right|
$$

and so the distance of the center $x_{0}$ of $B^{\prime}$ from $D_{2}$ is at most of the order of $\varepsilon r$ and then, by the arbitrariness of $\varepsilon$, we can also assume that $d\left(x_{0}, P\right) \leqslant \varepsilon r$. Moreover, the splitness of $B^{\prime}$ and the bound on the oscillation on each set $E_{i}$ imply that, in any way we take two points $x_{i} \in E_{i}$, we get

$$
\left|u\left(x_{1}\right)-u\left(x_{2}\right)\right| \geqslant \frac{\varepsilon^{-1}}{2} \sqrt{r} .
$$

Let $A=D_{1} \backslash p_{1}\left(K \cap B^{\prime}\right)$ and $a=r^{1-N} \mathcal{H}^{N-1}(A)$. By a trivial application of Fubini Theorem we get the following statement.

LEMMA 2.5. - There exists a positive constant c only depending on $N$, such that, with the above introduced notation, we have $a \leqslant c \varepsilon^{3}$. 
Proof. - By applying Fubini Theorem, we can find a chord $C$ orthogonal to $P$ which does not intersect $K$ and intersects the disks $D_{i}$, such that

$$
\int_{C}|\nabla u|^{2} \leqslant\left(\mathcal{H}^{N-1}(A)\right)^{-1} \int_{B^{\prime}}|\nabla u|^{2} \leqslant\left(a r^{N-1}\right)^{-1}(N+1) b_{N} r^{N-1}=a^{-1} c .
$$

Let $\left\{x_{i}\right\}=C \cap D_{i}$. By (2.11), (2.12) and by Hölder Inequality, we have

$$
\frac{\varepsilon^{-1}}{2} \sqrt{r} \leqslant\left|u\left(x_{1}\right)-u\left(x_{2}\right)\right| \leqslant \int_{C \cap F_{\varepsilon}}|\nabla u| \leqslant \sqrt{2 \varepsilon r}\left(\int_{C}|\nabla u|^{2}\right)^{1 / 2} \leqslant \sqrt{2 \varepsilon r a^{-1} c},
$$

from which the thesis easily follows.

Thus, if we set $D=P \cap B^{\prime}$ and we denote by $p$ the orthogonal projection onto $D$, since the difference between $\mathcal{H}^{N-1}(D)$ and $\mathcal{H}^{N-1}\left(D_{1}\right)$ is at most of the order of $\varepsilon^{2}$, we also get the following conclusion.

LEMMA 2.6. - If $B^{\prime}$ is as in Lemma 2.4 and $\varepsilon$ is sufficiently small, then

$$
\mathcal{H}^{N-1}\left(D \backslash p\left(K \cap B^{\prime}\right)\right)<\varepsilon r^{N-1} \text {. }
$$

A first consequence of Lemma 2.4 is the following.

LEMMA 2.7. - The ball $B^{\prime}$ found in Lemma 2.3 can be also taken in such a way that there exists a hyperplane $P$ such that

$$
r^{-1} \mathbf{d}\left(K \cap B^{\prime}, P \cap B^{\prime}\right)<\varepsilon
$$

Moreover $B^{\prime}$ can be also taken centered on $K$ and $P$ can be taken through the center of $B^{\prime}$.

The following result is a straightforward consequence of the semicontinuity of the functional with respect to the set variable $K$. The proof is an easy consequence of the results in [14], which can be applied under a normalization which sends $B$ into the unitary ball by a scaling operation. This is a particular case of a more general theorem which will be stated in [15] as Patching Theorem. For the sake of completeness, we sketch the proof of this particular case for which most of the technical devices employed in [15] are not needed and we refer the reader to [15] for more details which are missed or not fully developed in the following proof.

LEMMA 2.8. - For every $\varepsilon>0$ there exist $\delta>0$ and $R_{0}>0$ such that, if $K$ is $a$ minimum of $E$, if $B=B(x, R) \subset \Omega$ is such that $R \leqslant R_{0}$, and $P$ is a hyperplane through the center of $B$ such that $R^{-1} \mathbf{d}(K \cap B, P \cap B) \leqslant \delta$, then, set $B^{\prime}=B(x, R / 2)$, there exists a real function $v$ which is harmonic on $B^{\prime} \backslash P$ and such that

$$
R^{1-N} \int_{B^{\prime}}|\nabla u-\nabla v|^{2}<\varepsilon
$$




$$
R^{1-N}\left|\mathcal{H}^{N-1}\left(K \cap B^{\prime}\right)-\left(\frac{R}{2}\right)^{N-1} b_{N-1}\right|<\varepsilon
$$

Proof. - Assume by contradiction that the statement is false and take $\left(K_{n}\right)_{n \in \mathbb{N}}$ such that, after a normalization giving $R=1, \mathbf{d}\left(K_{n} \cap B, P \cap B\right) \rightarrow 0$. For every natural $n$, let $u_{n}=u\left(K_{n}\right)$, then $u_{n} \rightarrow u$ and $\nabla u_{n} \rightarrow \nabla u$ in $L^{2}(B)$, moreover it is easy to see that $\nabla u_{n}$ converges to $\nabla u$ uniformly on the compact subsets of $B \backslash P$. By the semicontinuity properties in [14], we have

$$
2^{1-N} b_{N-1} \leqslant \liminf _{n} \mathcal{H}^{N-1}\left(K_{n} \cap B^{\prime}\right) .
$$

If $2^{1-N} b_{N-1}<\limsup \operatorname{su}_{n} \mathcal{H}^{N-1}\left(K_{n} \cap B^{\prime}\right)$ or if $\nabla u_{n}$ does not converge to $\nabla u$ in $B^{\prime}$, then by adding to $K_{n}$, for a suitably small $a>0$, the set

$$
A=\left\{x \in \partial B^{\prime} \mid d(x, P)<a\right\},
$$

we get, for large $n$,

$$
\left|u-u_{n}\right| \leqslant a, \quad\left|\nabla u_{n}-\nabla u\right| \leqslant a
$$

in $\partial B^{\prime} \backslash A$. Let $\varphi_{n}$ be a harmonic extension of $u-u_{n}$ from $\partial B^{\prime} \backslash A$ on $B^{\prime}$, such that $\left\|\nabla \varphi_{n}\right\|_{L^{2}}$ is small. The substitution of $K_{n} \cap B^{\prime}$ by $\left(P \cap B^{\prime}\right) \cup A$ and of $u_{n}$ by $u-\varphi_{n}$, for large $n$, turns out to be convenient. The same argument proves that $u$ is harmonic.

A remarkable consequence of the previous lemma is the following statement, which allows the possibility to take $B^{\prime}$ with a small scaled Dirichlet integral.

LemmA 2.9. - For every $\varepsilon>0$, we can take the ball $B^{\prime}$ in Lemmas 2.3 and 2.7 in such a way to have

$$
\begin{gathered}
\mathbf{D}_{u}\left(B^{\prime}\right)<\varepsilon, \\
r^{1-N}\left|\mathcal{H}^{N-1}\left(K \cap B^{\prime}\right)-r^{N-1} b_{N-1}\right|<\varepsilon .
\end{gathered}
$$

Proof. - (2.17) can be trivially obtained by replacing $\varepsilon$ in Lemmas 2.3 and 2.7 by the constant $\delta$ correspondently given by Lemma 2.8 and by lowering by a factor $\frac{1}{2}$ the radius of $B^{\prime}$. The subharmonicity of $|\nabla v|^{2}$, where $v$ is as in Lemma 2.8, allows on the other side the proof of (2.16). Indeed, we take $\lambda<1$ and we consider the ball $B_{\lambda}$ having the same center of $B^{\prime}$ and radius $\lambda r$. For $\lambda=\varepsilon^{1 / N}\left(N b_{N}\right)^{-1}$, we have

$$
(\lambda r)^{1-N} \int_{B_{\lambda}}|\nabla v|^{2} \mathrm{~d} y \leqslant(\lambda r)^{1-N} \lambda^{N} \int_{B^{\prime}}|\nabla v|^{2} \mathrm{~d} y \leqslant \lambda N b_{N}<\varepsilon^{1 / N} .
$$

Since from (2.14) we also have

$$
(\lambda r)^{1-N} \int_{B_{\lambda}}|\nabla u-\nabla v|^{2} \mathrm{~d} y \leqslant \lambda^{1-N} r^{1-N} \int_{B^{\prime}}|\nabla u-\nabla v|^{2} \mathrm{~d} y \leqslant \varepsilon^{\frac{1-N}{N}}\left(N b_{N}\right)^{N-1} \varepsilon,
$$


from (2.18) we have $\mathbf{D}_{u}\left(B_{\lambda}\right) \leqslant c \varepsilon^{1 / N}$ which, by the arbitrariness of $\varepsilon$, proves the assertion. The other properties stated in Lemmas 2.3 and 2.8 are clearly preserved with the help of (2.11) when we pass from $B^{\prime}$ to $B_{\lambda}$, provided we take $\delta$ sufficiently small.

The results stated above enable us to prove the main theorem of this paper.

Proof of Theorem 1.1. - Let $\varepsilon>0, K$ be a minimum of $E$ and $B=B(x, R)$ with $x \in K$ and $R \leqslant 1$. Statements (1) and (3) follow by taking $B^{\prime}$ as given by Lemma 2.3; (2) and (6) follow from Lemma 2.9. By applying Lemma 2.7 we get assertion (5). By comparing (2) and Lemma 2.6 we deduce (4). Statement (7) follows by the application, allowed by (3) and (6), of the arguments of [4]. Finally, (8) follows by statement (6) and by the Hölder continuity of the function $u$ on both the hemispheres in which the ball is divided and (9) follows by (2.11) and by the arbitrariness of $\varepsilon$.

\section{REFERENCES}

[1] Ambrosio L., Compactness theorem for a special class of functions of bounded variation, Boll. Un. Mat. Ital. 3-B (1989) 857-881.

[2] Ambrosio L., Existence theory for a new class of variational problems, Arch. Rat. Mech. Anal. 111 (1990) 291-322.

[3] Ambrosio L., A new proof of the SBV compactness theorem, Calc. Var. 3 (1995) 127-137.

[4] Ambrosio L., Fusco N., Pallara D., Partial Regularity of Free Discontinuity Sets, II, Ann. Scuola Norm. Sup. Pisa Cl. Sci. (4) 24 (1997) 39-62.

[5] Ambrosio L., Fusco N., Pallara D., Functions of Bounded Variation and Free Discontinuity Problems, Clarendon Press, Oxford, 2000.

[6] Dal Maso G., Morel J.M., Solimini S., Une approche variationelle en traitement d' images: résultats d' existence et d' approximation, C. Rend. Acad. Sc. Paris, Série I 308 (1989) 549-554.

[7] Dal Maso G., Morel J.M., Solimini S., A variational method in image segmentation: existence and approximation results, Acta Mat. 168 (1992) 89-151.

[8] David G., $C^{1}$ arcs for the minimizers of the Mumford-Shah functional, SIAM J. Appl. Math. 56 (1996) 783-888.

[9] David G., Semmes S., On the singular set of minimizers of Mumford-Shah functional, J. Math. Pures Appl. 803 (1989) 549-554.

[10] David G., Semmes S., Fractured Fractals and Broken Dreams, Clarendon Press, Oxford, 1997.

[11] De Giorgi E., Ambrosio L., Un nuovo tipo di funzionale del calcolo delle variazioni, Atti Accad. Naz. Lincei Rend. Cl. Sci. Fis. Mat. Nat. s. 882 (1988) 199-210.

[12] De Giorgi E., Carriero M., Leaci A., Existence theorem for a minimum problem with free discontinuity set, Arch. Rat. Mech. Anal. 108 (1989) 195-218.

[13] Maddalena F., Solimini S., Concentration and flatness properties of bisected balls, Ann. Scuola Norm. Sup. Pisa (to appear).

[14] Maddalena F., Solimini S., Lower semicontinuity properties for functionals with free discontinuities, Arch. Rat. Mech. Anal. (to appear).

[15] Maddalena F., Solimini S., Blow-up techniques and regularity near the boundary for free discontinuity problems, in preparation.

[16] Morel J.M., Solimini S., Variational Methods in Image Segmentation, Birkhäuser, Boston, 1994. 
[17] Morrey C.B., Variational Multiple Integrals in the Calculus of Variations, Springer-Verlag, Heidelberg, New York, 1966.

[18] Mumford D., Shah S., Optimal approximation by piecewise smooth functions and associated variational problems, Comma. Pure Apple. Math. LXII-4 (1989).

[19] Rigot S., Big pieces of $C^{1, \alpha}$-graphs for minimizers of the the Mumford-Shah functional, Ann. Scoula Norm. Sup. Pisa Cl. Sci. 4 (2000) 329-349.

[20] Solimini S., Simplified excision techniques for Free Discontinuity Problems in several variables, J. Funct. Anal. 151 (1) (1997) 1-34. 